VII JORNADAS DE DIFUSIÓN DE LA INVESTIGACIÓN Y EXTENSIÓN - FCV-UNL

RESUMEN EXTENDIDO

\title{
CORRECCIÓN DEL DESPLAZAMIENTO ABOMASAL IZQUIERDO POR VIDEOLAPAROSCOPÍA
}

\author{
Leiva $\mathrm{CJM}^{1}$, Mazzini $\mathrm{R}^{1}$, Décima $\mathrm{M}^{1}$, Curiotti $\mathrm{J}^{1}$, Maggi $\mathrm{B}^{1}$, Kreczmann $\mathrm{E}^{2}$, \\ Repetto $\mathrm{A}^{3}$, Gonzales J3, Ranz Vallejo J ${ }^{4}$, Trucco $\mathrm{T}^{5}$ \\ ${ }^{1}$ Centro de Entrenamiento e Investigación en Cirugía Mini-Invasiva y Endoscopia (CEICME). UNL. \\ ${ }^{2}$ Cátedra de Cirugía II. FCV - UNL. Kreder 2805 (3080) Esperanza, Santa Fe \\ ${ }^{3}$ Cátedra de Clínica de Rumiantes. FCV - UNL. Kreder 2805 (3080) Esperanza, Santa Fe \\ ${ }^{4}$ Facultad de Veterinaria de la Universidad Complutense de Madrid, \\ ${ }^{5}$ Práctica privada.
}

* Correspondencia: Leiva CJM. E-mail: cristianjmleiva@yahoo.com.ar

Editado por: R. Sobrero, V. Matiller, Baravalle C.

CORRECTION OF LEFT DISPLACED ABOMASAL BY VIDEOLAPAROSCOPY.

SUMMARY.

Correction of left abomasal displacement by video laparoscopy. This article reviews the benefits of the laparoscopic abomasopexy used for correction and fixation of the left abomasal displacement in dairy cows. The first steps are similar to the conventional technique, with sedation, analgesia and antisepsis. Two small flank incisions were made, the first one is about 10 centimeters ventral to the lumbar transverse processes in the cranial aspect of the left paralumbar fossa, and the second one, more cranial than the other and is located $7 \mathrm{~cm}$ ventral to the transverse processes of the lumbar vertebrae in the left 11th intercostal space, these two are the entry points of instruments and optics. This technique allows us to shorten the surgical time, reducing the tissue exposure time, the possibility of intra and post-surgical infections and iatrogenic injuries. Therefore, we saw that the recovery time was shorter; and a quick return to production is guaranteed due to the low use of antibiotics.

Palabras clave: Desplazamiento abomasal izquierdo, laparoscopia, cirugía mini-invasiva.

Keywords: Left abomasal displacement, laparoscopy, minimally-invasive surgery.

El desplazamiento de abomaso es una patología, en la cual el órgano se desplaza de su ubicación normal, pasando por debajo del rumen, quedando entre éste y la pared costal izquierda, o sufriendo una torsión y ubicándose entre la pared costal derecha y su ubicación normal en el desplazamiento a la derecha. Se presenta especialmente al inicio de la lactancia, en sistemas de producción intensivos, donde la dieta suministrada posee cantidades elevadas de carbohidratos no estructurales a fin de elevar la producción láctea, estando asociadas a trastornos metabólicos, como hipocalcemia y cetosis (Albornoz et al., 2016). Los factores predisponentes y determinantes serian la acumulación de ácidos grasos volátiles e hipomotilidad, 
situación que provoca su dilatación y, en consecuencia, su desplazamiento a la izquierda o la derecha de la cavidad abdominal. El desplazamiento de abomaso a la izquierda es el más usual, representa entre 80 y $90 \%$ de los casos (Remsburg et al., 2011). Suele estar acompañado por una torsión leve del órgano, por lo que el tránsito del contenido abomasal se encuentra reducido y no totalmente bloqueado (Odriozola et al., 2009). Su condición puede durar varios días antes de presentar signos clínicos como anorexia, una abrupta disminución de la producción láctea, pérdida de peso, actividad ruminal reducida y cetosis secundaria (Albornoz et al., 2016). En ocasiones el desplazamiento abomasal puede pasar desapercibido (Coppock, 1974, Detilleux et al., 1997, Goff y Horst, 1997), representando entre el 60 y $70 \%$ de las patologías no diagnosticadas que ocurren entre las primeras 3 semanas de lactancia (Podetti, 2009). En algunos casos, si el desplazamiento llega a resolverse espontáneamente, esto determina un impacto económico negativo debido a la disminución de la producción láctea durante el periodo de convalecencia y costos originados por los tratamientos de los signos clínicos observados por el productor. Se ha reportado que, desde el parto hasta los 60 días pos diagnóstico, las vacas lecheras con este trastorno produjeron $557 \mathrm{~kg}$ de leche menos que los animales sanos, a su vez, los autores describen que el $30 \%$ de las pérdidas ocurrieron antes del diagnóstico en un estudio realizado sobre 12.572 vacas (Detilleux et al., 1997). Las tasas de mortandad son altas debido a que el diagnóstico certero de la patología se encuentra comprometido por falta de control y seguimiento en el periodo de posparto temprano. Si la patología no es diagnostica precozmente, trae aparejado mayores complicaciones cuando la resolución se realiza por métodos convencionales (Garnero y Perusia, 2002, Odriozola et al., 2009). Debido a estos inconvenientes se han desarrollado técnicas mínimamente invasivas (Janowitz, 1988, Christiansen, 2004), que disminuyen las complicaciones y consecuencias descriptas para los métodos convencionales (Garnero y Perusia, 2002); entre ellas podemos mencionar: recurrencias del desplazamiento, peritonitis local o difusa, dehiscencia de la herida, formación de fistula abomasal, mayor duración del procedimiento, disponibilidad de personal, mayores costos originados por los tratamientos, disminución en la producción láctea y descarte de leche debido al tiempo de retiro de los antibióticos utilizados (Newman, 2008). Las ventajas de las técnicas miniinvasivas para corregir estas patologías fueron descritas por numerosos autores (Mulon et al., 2006, Seeger et al., 2006, Wittek et al., 2009, Wittek et al., 2012): menor exposición y manipulación de los tejidos, menor tiempo quirúrgico, menor incidencia de infecciones posquirúrgicas, menor tamaño de las heridas quirúrgicas, (10 a 20 milímetros), menor tasa de recidiva, menor utilización de antibióticos y descarte de leche, disminución del dolor posquirúrgico, mayor producción láctea. Esto se apoya en las mediciones realizadas por los mismos autores como son: bajo recuento de leucocitos, eritrocitos y concentración del metabolito D-dimero en líquido peritoneal, menores niveles séricos de las enzimas proteína kinasa (PK) y lactato deshidrogenasa (LDH), mayor consumo de alimentos dentro de las 24 horas posquirúrgicas, menor tiempo en recuperar la motilidad abomasal y ruminal. En un esfuerzo por mejorar el tratamiento y los resultados de esta patología se describe una técnica miniinvasiva para la corrección del desplazamiento abomasal izquierdo en una vaca lechera, raza Holando Argentino, proveniente de un establecimiento comercial cercano a la facultad. El animal fue derivado al servicio del Hospital de Salud Animal, con diagnóstico presuntivo de desplazamiento de abomaso, lo cual fue confirmado, mediante examen clínico completo y sistemático del paciente, para luego llevar a cabo la técnica quirúrgica. Primero se realizó la sedación del animal, secundariamente se hizo la antisepsia del campo quirúrgico y luego se aplicó anestesia local subcutánea en forma de $L$ invertida. La técnica quirúrgica se basa en gran medida en la operación de Janowitz con modificaciones (Christiansen, 2004). El primer abordaje o portal se lleva a cabo en el centro de la fosa del ijar izquierdo a unos 10 centímetros $(\mathrm{cm})$ ventral de las apófisis transversas lumbares, donde con bisturí se realizó una pequeña incisión en la piel, para luego ingresar con un trocar Ternamian de 11 milímetros $(\mathrm{mm})$ (Karl Storz), esta vía nos permitió insertar una óptica de visión frontal Hopkins (0)), de 10 $\mathrm{mm}$ de diámetro y $40 \mathrm{~cm}$ de largo (Karl Storz), se conectó una cámara y fuente de luz (Karl Storz). En el mismo trocar se conectó la entrada de dióxido de carbono a la cavidad, con una bomba de insuflación (WEIST), llevando a lograr un pneumoperitoneo con una presión constante de $15 \mathrm{~mm}$ de mercurio, dicha maniobra nos permitió visualizar cómodamente el abomaso distendido entre la pared abdominal y el rumen. Para crear el segundo portal a la cavidad abdominal, se procedió de la misma manera que el primero pero el trocar Ternamian fue de $6 \mathrm{~mm}$ de diámetro (Karl Storz). El mismo se ubicó más craneal que el anterior, en el penúltimo espacio intercostal, aproximadamente 5 a $7 \mathrm{~cm}$ ventral de las apófisis transversas de las vértebras lumbares. A través del acceso craneal, se ingresó al abomaso, en su punto más alto, con un trocar (instrumento para la fijación de toggles, Karl Storz) de $30 \mathrm{~cm}$ de largo e inmediatamente se introdujo una sutura de palanca, Toggle (pasador de acero de $4 \mathrm{~cm}$ de largo con doble hilo central fijo de 80 $\mathrm{cm}$ de largo) (Karl Storz). Luego de conectada la sutura, se esperó a que se elimine todo el gas del abomaso a través del trocar, logrando que el órgano se ubique en el piso de la cavidad. Una vez retirado el fijador de Toggle, los dos hilos de Toggle quedaron en el exterior a través del trocar, fueron fijados con una pinza hemostática, de modo que no puedan deslizarse hacia la cavidad abdominal. Por el acceso craneal, se ingresó con un instrumento de $90 \mathrm{~cm}$ de largo, hueco, con un diámetro de $8 \mathrm{~mm}$, ligeramente curvado y engrosado en su extremo distal (endo-oliva), de modo que no haya riesgo de lesiones (Karl storz). Se dirigió hacia abajo de 
la cavidad abdominal, avanzando con su curvatura hacia la línea media. Este procedimiento no puede seguirse endoscópicamente, pero es fácil de controlar desde el exterior con la mano libre del cirujano. El extremo se puede tocar palpando en la zona hipocondriaca derecha (ubicación normal del abomaso). Una vez ubicado el lugar correcto, se introdujo dentro un estilete puntiagudo y en forma de aguja, con el cual se perforó la pared abdominal hasta el exterior. Luego un asistente enhebro un hilo de nylon de 1 metro de largo a través del ojo del estilete, que nos sirvió de guía. El estilete se retrae hacía afuera con el hilo lentamente hasta el trocar de acceso. Un extremo del hilo guía permanece ventral al abdomen y el otro extremo en acceso del trocar craneal. Desde el acceso, quedaron colgando tres hilos, dos provienen de la sutura palanca (Toggle) desde el abomaso y uno proviene del sitio de fijación posterior en la pared abdominal ventral. Estos tres hilos se anudaron juntos. Luego, el asistente traccionó del extremo ventral del hilo guía hasta que aparezca este nudo, y con ello se trasladó el abomaso a su zona de ubicación normal. Con ambos hilos de Toggle se realizó un nudo, interponiendo un rollo de gasa entre éste y la pared abdominal. Dicha técnica nos permitió acortar los tiempos de cirugía, disminuyendo el tiempo de exposición de los tejidos, la posibilidad de infecciones durante y post cirugía y lesiones iatrogénicas. Por lo cual el tiempo de recuperación fue menor y un rápido retorno a la producción. Los resultados obtenidos sugieren que se justifica la inversión en equipamiento y formación en esta técnica, para ser aplicada en sistemas actuales de producción.

\section{Bibliografía}

Albornoz L, Albornoz JP, Morales M, Fidalgo LE. 2016. Hipocalcemia puerperal bovina. -Revisión. Veterinaria (Montevideo) Volumen 52 № 201. 28-38.

Christiansen K. 2004. Laparosckopisch kontrollierte operation des nach links verlagerten Labmagens (Janowitz-operation) ohn Ablegen des Patienten. Tiera“ rztl Praxis; 32:118-21.

Coppock CE. 1974. Displaced Abomasum in Dairy Cattle: Etiological Factors. Symposium: health and management practices for high production. Department of Animal Science Cornell University Ithaca, New York 14850

Detilleux JC, Grohn YT, Eicker SW, Quaas RL. 1997. Effects of Left Displaced Abomasum on Test Day Milk Yields of Holstein Cows. J Dairy Sci 80:121-126

Goff JP, Horst RL. 1997. Physiological Changes at Parturition an Their Relationship to Metabolic Disorders. J Dairy Sci 80:12601268

Garnero. O, Perusia. O. 2002. Manual de anestesias y cirugías del bovino. Pp. 41-46, Cap. 3, $2^{\circ}$ Edición
Janowitz H. 1988. Laparoskopische Reposition und Fixation des nach links verlagerten Labmagens beim Rind. Tierärztl Prax. 26 (G): 308-13.

Newman KD, Harvey D, Roy JP. 2008. Minimally invasive field abomasopexy techniques for correction and fixation of left displacement of the abomasum in dairy cows. Vet Clin Food Anim 24. 359-3829

Mulon PY, Babkine M, Desrochers A. 2006. Ventral laparoscopic abomasopexy in 18 cattle with displaced abomasum. The American College of Veterinary Surgeons 0161-3499/06 doi:10.1111/j.1532-950X.2006.00156.x

Odriozola E, Campero CM, Cantón GJ, Licoff N. 2009. "Descripción de un caso de desplazamiento abomasal derecho en vacas lecheras en argentina". Rev Med Vet (B. Aires) 90(34): $45-48$.

Podetti M V. 2009. El desplazamiento de abomaso: ¿Cuánto nos afecta? Producir XXI, Bs. As., 17(209):46-52.

Remsburg W, Galligan DT, Ferguson JD. 2011. "Use of decision analysis to evaluate the delivery method of veterinary health care on dairy farms as measured by correction of left displaced abomasum". J Am Vet Med Assoc 238(1): 60-65.

Seeger T, Kümper H, Failing K, Nat R, Doll K. 2006. Comparison of laparoscopic-guided abomasopexy versus omentopexy via right flank laparotomy for the treatment of left abomasal displacement in dairy cows. AJVR, Vol 67, No. 3.

Wittek T, Locher LF, Alkaassem A, Constable PD. 2009. Effect of surgical correction of left displaced abomasum by means of omentopexy via right flank laparotomy or two-step laparoscopy-guided abomasopexy on postoperative abomasal emptying rate in lactating dairy cows. J Am Vet Med Assoc.; 234:652-657.

Wittek T, Fürll M, Grosche A. 2012. Peritoneal inflammatory response to surgical correction of left displaced abomasum using different techniques. Veterinary Record. doi: 10.1136/vr.101107 